We also suggest that instead of selecting eight cells out of 1,000 for analysis, one should show the frequency distribution of grains over as many of the cells as is practical. The unlabelled cells are as valid an experimental sample as the presumably "labelled" ones and excluding them from the analysis is a biased sampling procedure. A bacterial ${ }^{3} \mathrm{H}-\mathrm{RNA}$ control and a poly A competition are also necessary for proper analysis of these results.

Department of Pathology,

W. Prensky

Sloan Kettering Institute,

New York, NY 10021

Department of Biological Chemistry,

Harvard Medical School,

Boston, Massachusetts 02115

Received July 10, 1972.

${ }^{1}$ Price, P. M., Conover, J. H., and Hirschhorn, K., Nature, 237, 340 (1972).

2 Bishop, J. O., Pemberton, R., and Baglioni, C., Nature New Biology, 235, 231 (1972).

3 Wimber, D., and Steffensen, D. M., Science, 179, 639 (1970).

\section{Do Bacteria Have a Nuclear Membrane?}

THE idea of Stanier and van Niel ${ }^{1}$ that bacteria (and possibly blue-green algae) are "prokaryotic" organisms, lacking a nuclear membrane, appears to have been accepted almost without argument. All other cells possess such a membrane, and are "eukaryotic", according to this theory.

The inner layer of the bacterial cell envelopes, however, which is commonly regarded as comparable with the semipermeable membrane of other cells, carries the attachments of the flagellum and the nuclear system. In "eukaryotic" protista it is with the nuclear membrane that such structures are associated $^{2}$.

Bacterial murein ${ }^{3}$ has been described ${ }^{4}$ in this journal as a layer between the cell wall proper and the cytoplasmic membrane; "the meat in the sandwich", which most appropriately consists of a lipoprotein complex. Although this may, and probably does, differ in chemical detail from the composition of classical cell membranes, and it is not alike in all bacteria, above the membrane, which is performing two of the most important known functions of the primitive nuclear membrane, there is a structure that, in some cases, at least chemically, suggests a membrane more than a cell wall, and could fairly be regarded as having been evolved from a previously existing, semipermeable membrane.

I suggest on the basis of general morphological relationships 5 that the structure which now represents the cell membrane of bacteria may have originated in the nuclear membrane of an ancestral form; with the corollary that the murein layer could be derived from the cell membrane, the functions of which had been taken over by the nuclear membrane. The differences which may now exist between the composition of these organs, and that of their hypothetical forerunners, are no greater than those of many comparable examples, in the wider field and longer scale of evolution (for example, the retinal rods from cilia).

There is no reason to presume that bacteria, because of their small size, are in any way primitive ${ }^{6}$. They are, indeed, highly specialized organisms, making use of their small size, and consequent low bulk-surface ratio and rapid metabolism. They are almost certainly derived from other, more primitive, flagellate protista, and the origins of their organs must be sought in the general structural pool.

This hypothesis gives rise to further questions; the most obvious of these is: what service is such a transformation, whereby the cell membrane reinforces the wall, while the nuclear membrane takes over its function? The best available answer refers back to the advantages of a small size and a large surface area, as the latter can be maintained, against the relatively strong forces of surface tension, in an already exceptionally small cell, only by great rigidity in the cell envelopes.

It may be that the supposed resemblance between bacteria and blue-green algae in having no nuclear membrane will prove to be as invalid as the previous theory; that they were alike in having no nucleus at all. They are alike mainly in their small size, and in the convergent adaptations that this produces. Positive groupings, based on negative criteria, are seldom durable in biology, and separate creation, even of organs, must have some evolutionary background.

\section{Department of Bacteriology, \\ The Medical School, \\ University of Birmingham, \\ Birmingham, B15 2 TJ}

Received June 16; final revision October 12, 1972.

'Stanier, R. Y., and van Niel, C. B., Arch. Mikrobiol., 42, 17 (1962)

Bisset, K. A., Bild Wiss., 7, 212 (1970).

3 Braun, V., and Bosch, V., Proc. US Nat. Acad. Sci., 69, 970 (1972).

4 Editorial, Nature, 237, 311 (1972).

5 Bisset, K. A., The Cytology and Life-History of Bacteria, third edition, 72 (Livingstone, Edinburgh, 1970).

6 Bisset, K. A., Vistas in Botany, 1, 313 (Pergamon Press, Oxford, 1959).

\section{Can Mitochondrial Complementation be Used as a Tool in Breeding Hybrid Cereals?}

SEVERAL papers ${ }^{1-6}$ have been published in which it is claimed that mitochondrial heterosis and mitochondrial complementation are related to the yield potential of $F_{1}$ hybrid cereal cultivars. Mitochondrial heterosis is measured by the superior oxidative and phosphorylating efficiency of mitochondria isolated from heterotic $F_{1}$ hybrid cultivars when compared with those isolated from the corresponding parental cultivars. Although these parameters can be measured only after a small quantity of $F_{1}$ hybrid seed has been produced they may prove useful to plant breeders as a preliminary appraisal of the yield potential of an $F_{1}$ hybrid before undertaking extensive field trials.

Mitochondrial complementation is a more complex phenomenon, reported to occur when mitochondria isolated from the two prospective parents are mixed in vitro. The phenomenon can be recognized by the superior biochemical activity (measured by the rate of oxidation and efficiency of phosphorylation) of a $1: 1$ mixture of the mitochondria of the two potential parents over the arithmetic mean of the two preparations when assayed separately. Sarkissian and Srivastava ${ }^{7}$ claim that there is a correlation between mitochondrial complementation and mitochondrial heterosis in $\mathrm{F}_{1}$ hybrid maize cultivars and have extended their observations to include $F_{1}$ hybrid wheat ${ }^{3}$. Mitochondrial complementation is therefore a potentially much more useful tool in plant breeding programmes than mitochondrial heterosis because it would predict, without first producing the hybrid, which cultivars, when crossed, would yield heterotic $F_{1}$ hybrids.

$\mathrm{McDanie}^{6}$ reiterates that both mitochondrial heterosis and complementation can be used to predict the relative yield potentials of $F_{1}$ hybrid barley cultivars. He states that use of mitochondrial complementation "should provide a more efficient and rapid method of screening potential breeding material and evaluating hybrids". However, McDaniel does not discuss the relative merits of complementation and heterosis 